\title{
Modeling Capacitive Coupling Systems for Body Coupled Communications
}

\author{
Ricardo J.S. Matias \\ IEETA - rjsm@ua.pt
}

\author{
Manuel Bernardo Cunha \\ IEETA - mbc@det.ua.pt \\ Rui Manuel Martins \\ IEETA - rmm@ua.pt
}

Alexandre Manuel Mota

IEETA - alex@ua.pt

\begin{abstract}
With the exponential grow of personal wireless devices, issues like power consumption, communication security and interference immunity are gaining more and more importance. Body Area Networks (BAN), due to their reduced range, are by nature relatively low power, but as an answer to achieve even a more confined range, Near Field Communications (NFC) and Body Coupled Communications (BCC) have been proposed. While the first is used to link two devices that are physically very close, the BCC concept broadens the communication range to the region around the human body. One of the possible technologies supporting BCC is based upon relatively low frequency capacitive coupling between emitter and receiver. Understanding the connection between the physical layout of an electromagnetic system and its electrical model is important to understand its performance and critical aspects. However, although much information exists about antennas and their radiated energy, very few work has been done on how energy is transferred between the electrodes used in a capacitive communication system. In this paper we propose a simple model for estimating the gain of a capacitive coupling system. This model has been validated by $3 \mathrm{D}$ electromagnetic simulations and double checked with practical experiments performed in a controlled environment (faraday cage with only the minimum indispensable instrumentation equipment). The results are similar within an order of magnitude which, for the intended use of the model, proves to be accurate enough.
\end{abstract}

Keywords - Body Area Network (BAN), Capacitive Coupling, Near Field, EM - Electromagnetic Fields, Low Power.

\section{INTRODUCTION}

When Wireless Communications were invented their use was primarily to make long distance communications, typically to broadcast Radio and TV. The interest in the electromagnetic fields was always concerning to radiated or far field systems. However, with the huge evolution in portable electronic gadgets the nowadays crowded Electromagnetic Spec-

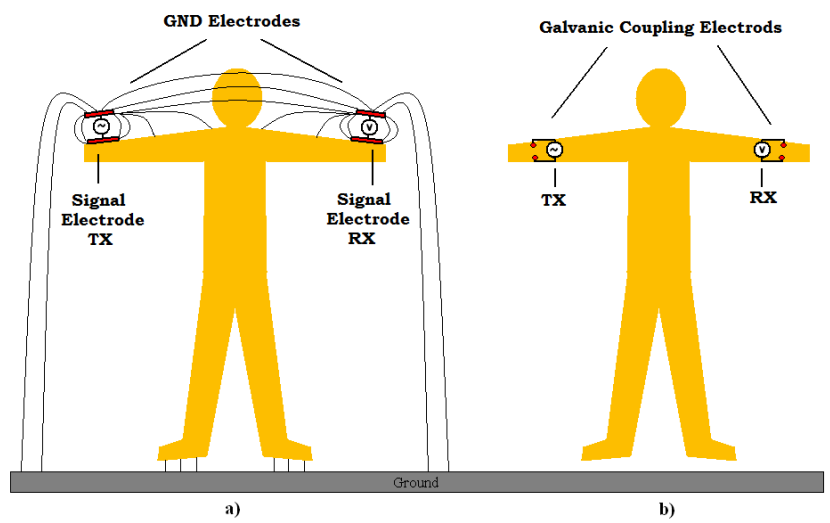

Figure 1: a)Capacitive coupling communication system using the human body vs. b) Galvanic Coupling Intrabody communication.

trum is demanding for simple, low power, secure and interference immune communications for the ever-growing market of Personal Area Networks (PAN) and Body Area Networks (BAN). An example of such networks is based upon Body Coupled Communications (BCC) [1] typically used (but not limited) to monitor biological signals. It is in this context that Reactive Coupled electromagnetic systems, like Capacitive/Inductive Coupled systems, come to play. Near Field Communication (NFC) is such a case where inductive coupling is used [2], but this work concerns capacitive coupling communications first reported in [3] and presented in Figure 1a). Other strategies to accomplish such a network are Mechanical Osteo communication [7] and Galvanic Coupling Intrabody communications [6] (see Figure 1b). The later has advantages when considering intrabody communications (e.g. in devices implanted inside the human body), but requires conducting electrodes which are more complex and expensive. Typical conductive electrodes are of wet type (mainly $\mathrm{Ag}-\mathrm{AgCl}$ ) and cannot be used for long periods. For non-medical applications, conductive electrodes require devices in contact with skin, however capacitive ones can be held on the top of the clothes, e.g. inside pockets. Therefore for long time/permanent vital signal monitoring or for nonmedical applications we consider capacitive communication a better solution.

BCC are simultaneously power consumption friendly and very secure (as they are linked by the human body and reach 
only few decimeters from the body surface). This very well confined range is not possible to obtain with radiating technologies like Zigbee or Bluetooth. On the down side of BCC we have the low transmition rate ([3] achieved few kbps). Although the ultimate requirements for a general purpose BAN demand for multimedia capacity [8] there are many useful applications requiring low bit rates. Anyhow, in [5] a transmition rate of $10 \mathrm{Mbps}$ is reported, but the power consumption is high.

The reasons why $\mathrm{BCC}$ are more secure (in terms of space range definition) and consume potentialy less to communicate than a conventional $\mathrm{RF}$ radiating system are related to the amount of energy involved in communication and the distribution of the transmitter energy in space. In the case of a BCC, only the small amount of the energy used for communication is transfered between transmitter and receiver (reactive coupling). However, for a radiating system, the total energy used in the communication system is lost, even if there is only a transmitter and no receivers! In BCC, energy is also almost reactive, because for lower frequencies BCC is a Lumped Parameter System, and Lumped Parameter Systems usually radiate very little. In the case of a radiation system, the energy goes away through the infinity, but the electric field decays more abruptly in a Capacitive Coupling system. For example, in the case of a static charge dipole (see Figure 2), the electric field decay is proportional to $d^{-3}$ (with $d$ being the distance to the dipole center) [14], whereas in an antenna considering the far field region, it decays with $d^{-1}$ [17] (in terms of energy the decay ratio is proportional to $d^{-6}$ and $d^{-2}$, respectively [16]).

As the interest in capacitive coupled communications is recent, very few work has been reported in literature, namely about channel modeling, the main subject of the next section, which also presents results from simulations that verify the quality of the theoretical results. Then the paper follows with a section where practical experiments are compared with simulations to double check the accuracy of the previous results. Finally in section 4 some conclusions are drawn.

\section{MODELING THE CHANNEL OF A CA- PACITIVE COUPLED COMMUNICATI- ON SYSTEM}

Some of the few work reported in the literature regarding BCC channel modeling [11] [12] consider only the grounded case using setups based on standard measurement equipment which is strongly coupled to the earth ground. This

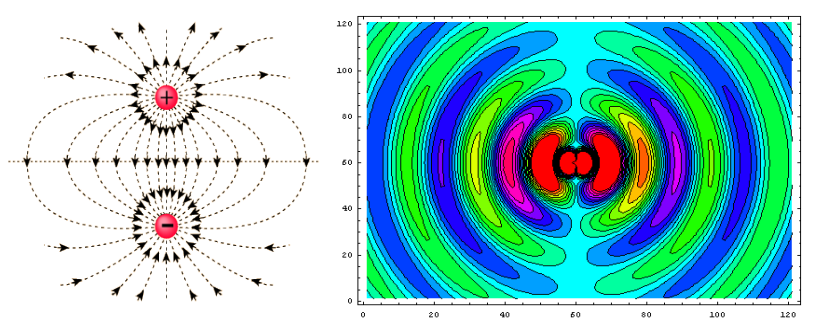

Figure 2: Static Dipole vs. Radiating Dipole [18]. scenario changes the real conditions of capacitive coupled communications and therefore produces unreliable results [4]. In [3][4] correct setups were used, but in both cases only experimental results are given. [4] reports extensive results for different frequencies, sizes and other parameters, but do not presents a theoretical model.

In order to look for a simple channel model for the capacitive coupling communication systems using the human body, it is necessary to simplify all aspects of the general model (in Figure 1a) that do not compromise seriously the accuracy of the results. It is assumed that the capacitance between the lower plates of the emitter and receiver electrodes (whatever their shape is) will be much larger than the capacitance between their top plates (a detail of an electrode is shown in Figure 3), which results in considering that the lower plates are short-circuited to the human body (Figure 4). As at low frequencies (less than $10 \mathrm{MHz}$ - far bellow $2.4 \mathrm{GHz}$ used in Bluetooth, Wi-Fi and Zigbee [18]) the human body can be regarded as being a good conductor ("for an adult male 2 meters tall and an average diameter of 0.3 meters, with an average resistivity of $10 \mathrm{ohm}$-meter, a resistance of 251 ohms is calculated") [3] and also relatively planar (which is arguable, but necessary to obtain the desired simple mathematics), the model displayed in Figure 1a) can be simplified for a limit case to the system depicted in Figure 5, which is described in electrostatics by a network of capacitances as illustrated in Figure 6. This is true if the distance between the body and the electrodes is much smaller than that between electrodes (fortunately this is the normal case even if the electrodes lay on the top of the clothes).

\subsection{Theoretical Model}

The electrostatics of every system of conductors can be described by a set of equations, that relate charges to electric potentials trougth (let's say) capacitance coeficients [14]. It is possible to represent them (for our case) with an electric circuit as displayed in Figure 6, which for low frequencies is a good (quasistatic) aproximation. Assuming that emitter and receiver electrodes are physically equal, the load capacitances $\left(C_{L}\right)$ have the same value (and for reasonable sized electrodes and distance between them) then $C_{L} \gg C_{c o u p l}$ $\left(C_{\text {coupl }}\right.$ - Coupling Capacitance). The electric capacitance

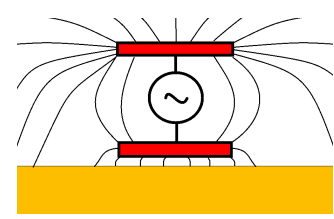

Figure 3: Detail of a capacitive electrode.

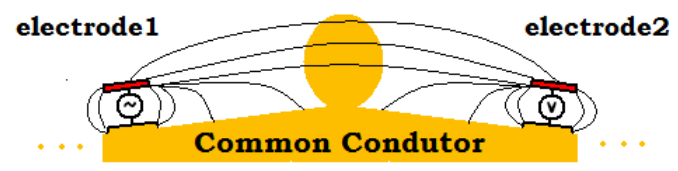

Figure 4: Simplified view of a capacitive coupling communication system using the human body. 
is the ratio of charge per potencial diference, so the capacitances of the system are:

$$
\begin{aligned}
C_{L} & =\frac{q_{L 1}}{V_{1}}=\frac{q_{L 2}}{V_{2}} \\
C_{\text {coupl }} & =\frac{q_{a}}{V_{1}-V_{2}}
\end{aligned}
$$

Let the net charge of the right conductor be zero $\left(q_{2}=0\right)$. We have that:

$$
\begin{aligned}
& q_{1}=q_{L 1}+q_{a} \\
& q_{2}=q_{L 2}-q_{a} \Leftrightarrow q_{a}=q_{L 2}
\end{aligned}
$$

As $C_{L} \gg C_{\text {coupl }}\left(q_{1} \simeq q_{L 1}\right)$, we can simplify:

$$
C_{L} \simeq \frac{q_{1}}{V_{1}}
$$

Let $V_{2 b y 1}$ be the potencial that apears in 2 when we apply a potencial $V_{1}$ in conductor 1 . We have:

$$
C_{\text {coupl }}=\frac{q_{a}}{V_{1}-V_{2 b y 1}}
$$

Because $V_{2 b y 1} \ll V_{1}$ :

$$
C_{\text {coupl }} \simeq \frac{q_{a}}{V_{1}}=\frac{q_{L 2}}{V_{1}}=\frac{C_{L} \cdot V_{2 b y 1}}{V_{1}}
$$

So, when $C_{L} \gg C_{\text {coupl }}, q_{2}=0$ :

$$
\frac{V_{2 b y 1}}{V_{1}} \simeq \frac{C_{\text {coupl }}}{C_{L}}
$$

which is exactly what is expected from simple circuit analisys (capacitive impedance divider).

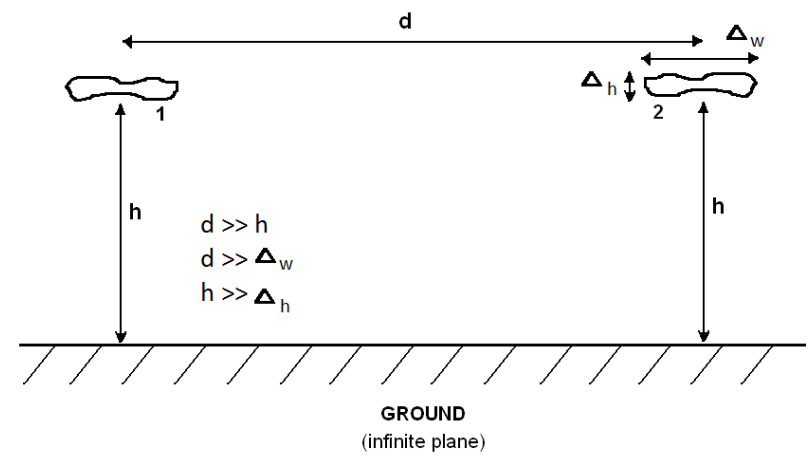

Figure 5: Three conductor system with the third conductor as an infinite plane.

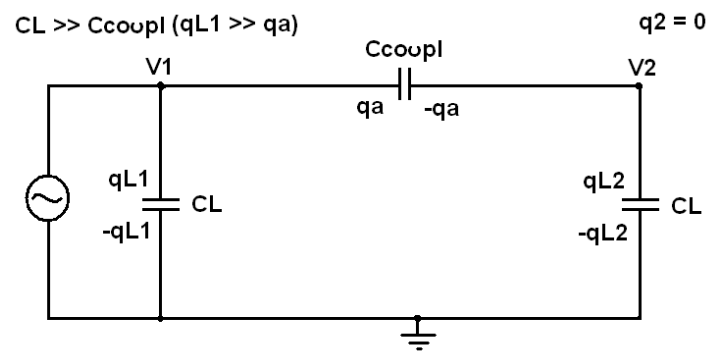

Figure 6: Three condutor system capacitive coupling equivalent electric circuit.
If the dimensions of the electrodes are those of Figure 5: $d \gg h ; h \gg \Delta h ; d \gg \Delta d$ (the electrodes are thin and perfectly planar metal plates), it can be assumed for the sake of electric field calculations, that charges can concentrate in points. With this simplification in mind, the value of $V_{2 b y 1}$ can be calculated and then used to determine the value of $C_{\text {coupl }}$ (as function of $C_{L}$ ). Making a mirror with the infinite plane, there is an image of the charge on the left conductor as in Figure 7. The transmitter can be understood as putting a dipole charge at some height and the receiver as an electric field sensor. So one can say that for the emitter is good to have a larger $C_{L}$ (more charge give more electric field at receiver) and for both emitter and receiver a large height! It is to note that the receiver do not influence the emitter because the transferred energy is very low compared to the reactive energy of emitter! Generically, from coulomb's law we have:

$$
\begin{aligned}
& V=k_{e} \cdot \frac{q}{r} \\
& k_{e}=\frac{1}{4 \pi \epsilon_{0}}
\end{aligned}
$$

with $r$ the distance from the charge.

Therefore, taking Figure 7 in consideration, we obtain:

$$
V_{2 b y 1}=k_{e} \cdot \frac{q_{1}}{d}-k_{e} \cdot \frac{q_{1}}{\sqrt{(2 h)^{2}+d^{2}}}
$$

Relating the value of charge through the capacitance of conductor 1 ,

$$
V_{2 b y 1}=V_{1} \cdot k_{e} \cdot C_{L} \cdot\left(\frac{1}{d}-\frac{1}{\sqrt{(2 h)^{2}+d^{2}}}\right)
$$

Replacing (12) in equation (8) results:

$$
\frac{C_{\text {coupl }}}{C_{L}} \simeq k_{e} \cdot C_{L} \cdot\left(\frac{1}{d}-\frac{1}{\sqrt{(2 h)^{2}+d^{2}}}\right)
$$

This formula models the voltage gain as function of the load capacitance and the system dimensions! Note that when the conductor's thickness is not negligible, the effective height of the dipole charge on emitter and the effective potential received cannot be exactly known. Actually, the experimental tests seem to show that when the common conductor

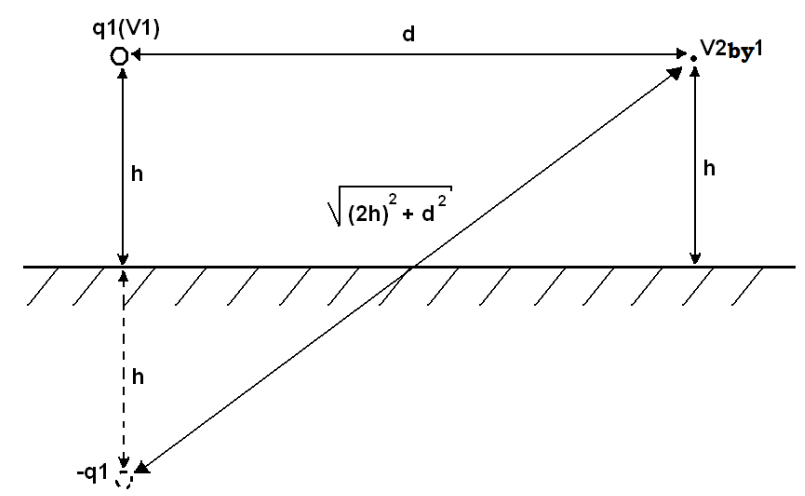

Figure 7: Electric potential calculation of a point charge and an infinite conductive plane. 
is smaller and convex, the effective gain of the system increases! Remembering that typically $d \gg h$ and considering (15), then (13) simplifies to:

$$
\begin{gathered}
\frac{C_{\text {coupl }}}{C_{L}} \simeq 2 \cdot k_{e} \cdot C_{L} \cdot\left(\frac{(h)^{2}}{(d)^{3}}\right) \\
\frac{1}{d}-\frac{1}{\sqrt{(2 h)^{2}+d^{2}}} \simeq \frac{\sqrt{d^{2}}+\frac{(2 h)^{2}}{2 d}-d}{d \sqrt{(2 h)^{2}+d^{2}}}
\end{gathered}
$$

\subsection{Simulations}

For electromagnetic simulations, Ansoft HFSS (a 3D Full Wave Electromagnetic Simulator) [19] and SAP - Smart Software Package that has a simulator called STAP for static coupling capacitances extraction [20] were used. With Ansoft HFSS, a lumped parameter simulation of the system (bounded by a faraday cage) at given frequency was performed, which returned a $Z$ parameters matrix. With the $Z$ parameters of the two-port network that Ansoft HFSS calculates, using the equivelent circuit of Figure 8, it is possible to derive $Z_{L}$ and $Z_{\text {coupl }}$ (and therefore $C_{L}$ and $C_{\text {coupl }}$, with the assumption of an almost reactive circuit).

$$
\begin{gathered}
{\left[\begin{array}{l}
V_{1} \\
V_{2}
\end{array}\right]=\left[\begin{array}{ll}
z_{11} & z_{12} \\
z_{21} & z_{22}
\end{array}\right] \cdot\left[\begin{array}{l}
I_{1} \\
I_{2}
\end{array}\right]} \\
z_{11}=z_{22}=Z_{L} \cdot \frac{Z_{\text {coupl }}+Z_{L}}{2 Z_{L}+Z_{\text {coupl }}} \\
z_{12}=z_{21}=\frac{Z_{L}^{2}}{2 Z_{L}+Z_{\text {coupl }}}
\end{gathered}
$$

We could simplify this for $Z_{\text {coupl }} \gg Z_{L}$ :

$$
\begin{aligned}
Z_{L} & =z_{11} \\
Z_{\text {coupl }} & =\frac{z_{11}^{2}}{z_{12}} \\
C & =\frac{1}{2 \pi f|Z|}
\end{aligned}
$$

With this information it is possible to perform simulations and compare the values of channel gain to our theorical approximation (using the simulated load capacitance - $C_{L}$, derived from averaged $Z_{L}$ ).

\subsection{Simulation Results}

A large number of simulations for several different electrode parameters and distances (see Figure 9) has been performed. The main parameters and distances are:

$e$ - electrode's width (square shape)

$a$ - wings' height (this parameter is explained below)

$h$ - electrode's height (above the ground plane)

$l_{f c}$ - faraday cage width (cubic shape)

$f$ - frequency

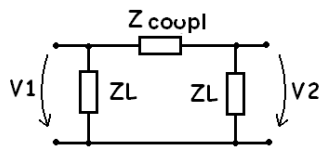

Figure 8: 2-port network ( $\pi$ representation).
Figure 10 presents the results for simple electrodes (like those depicted in Figure 12) at a frequency of $12.6 \mathrm{MHz}$. Taking Figure 9 in consideration, this means that $a=0$. The

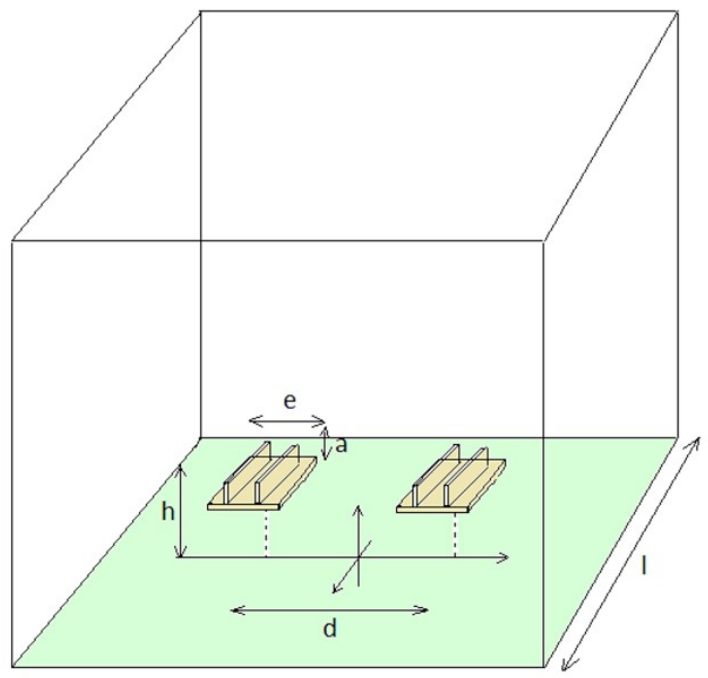

Figure 9: Electrode parameters.
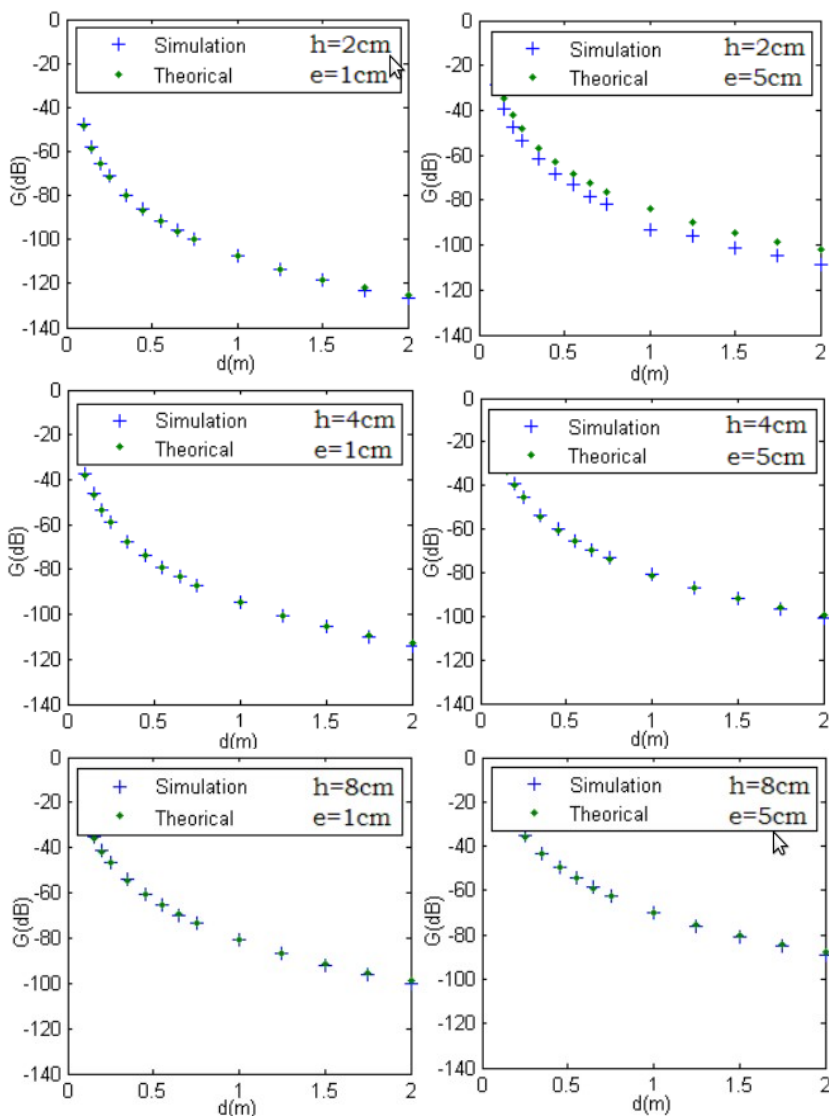

Figure 10: Capacitive coupling channel gain HFSS simulations vs. Theoretical approximations $\left(l_{f c}=\right.$ $6 m, f=12.46 \mathrm{MHz}, a=0)$. 


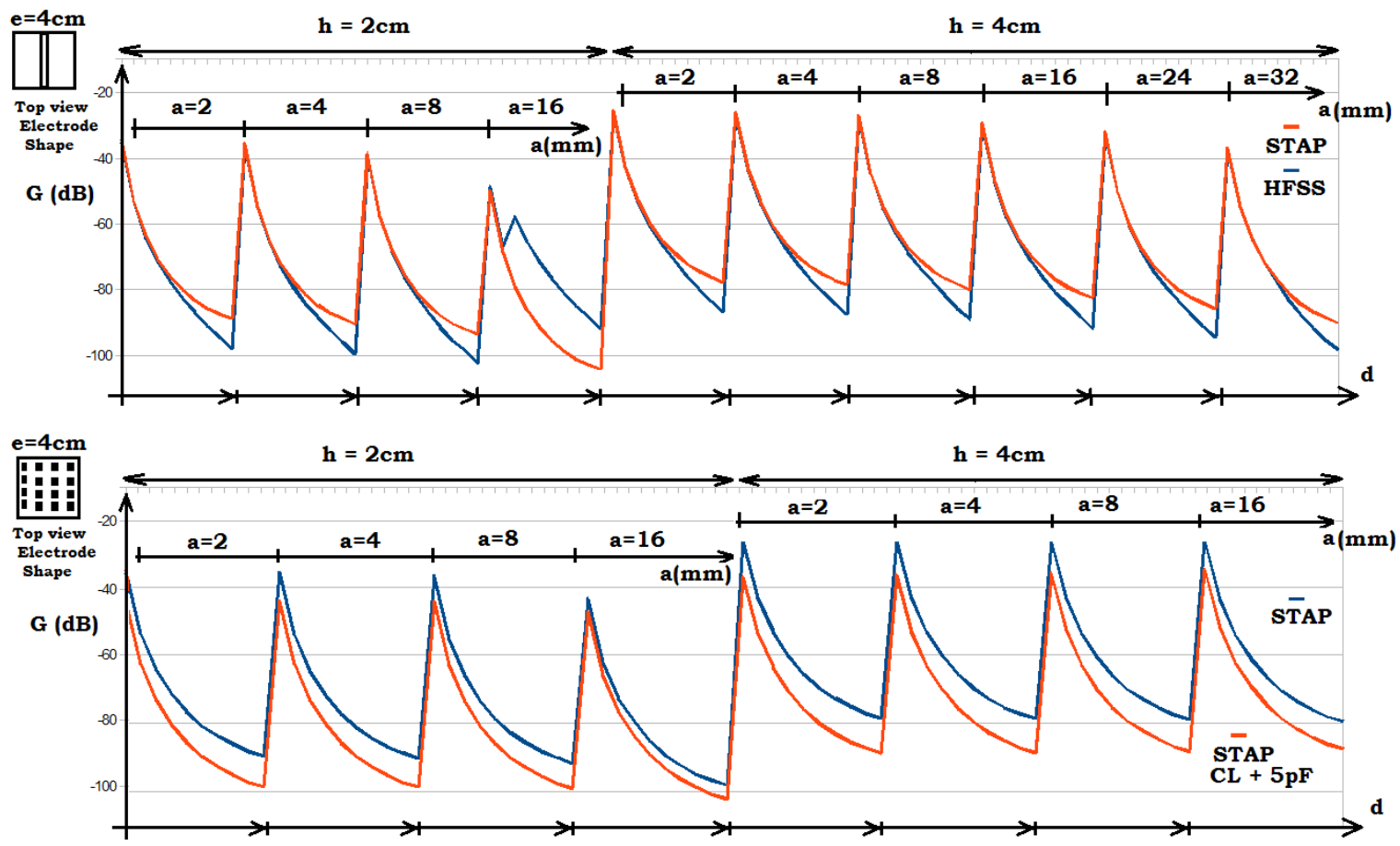

Figure 11: Capacitive coupling channel gain simulations varying electrode's wings $\left(l_{f c}=2.5 \mathrm{~m}, f=12.46 \mathrm{MHz}\right.$ (HFSS)).

voltage gain error between the theoretical model and simulation results is generally less that $3 \mathrm{~dB}$ (except for $\mathrm{h}=2 \mathrm{~cm}$ and $\mathrm{e}=5 \mathrm{~cm}$, which seems a simulation's convergence fault). The results improve with larger electrode's area and height. Of course, for practical reasons both area and height cannot be too large.

After simulating perfectly plane electrodes, another set of simulations was performed considering electrodes with wings (see Figure 9 again). The authors considered that wings should improve the electrode's performance. To make comparisons fare, the total height of these electrodes included the wing's height. But as observed in Figure 11 the results were not the expected and it seems that is always better to have a simple planar electrode at the maximum possible height. This happens for simple wings (top results) as well for electrodes with an array of many small "parallelepiped" wings (bottom results). Figure 11-top presents simulations performed both by HFSS and STAP (electrostatic simulator). The results look very similar with exception for large distances between electrodes. This difference can be due in STAP no metal cage has been considered, although the simulation domain was exactly the same. The results in Figure 11-bottom are coming only from STAP simulations (as their solid modeling tool has a simple way to create the array of small "parallelepiped" wings). The second curve considers the effect of the input capacitance of the electronics connected to the receiver electrode. But, once more it looks that the wing effect does not pay the extra effort needed.

\section{PRACTICAL EXPERIMENTS}

Although the degree of confidence in the simulators were high, their performance was double checked comparing si- mulation results against practical experiments. In order to unambiguously set the boundary conditions of the experiments, it was decided to perform the tests inside a Faraday cage, as done with the HFSS simulator. This also made tests easier, as they were performed in an interference free environment.

Figure 12-left shows the electrodes used in the practical setup (the top electrode height is $8.6 \mathrm{~cm}$ from the bottom conductor), and (on the right) the layout of HFSS simulations (that took into account both the electrodes, the metal screws to fix them and the bottom conductor). In the tests a portable oscilloscope and signal generator were used, and to overcome the oscilloscope lack of sensitivity, voltage gain has been added to the transmitter (signal generator) with a $\mathrm{RF}$ transformer. The equipment connects the electrodes via coaxial cables layered on the top of the metal floor of the faraday cage in order to minimize their influence in the circuit! For the same reason the instruments were positioned near the lower corners of the faraday cage. With these procedures, the setup approaches the conditions that led to the
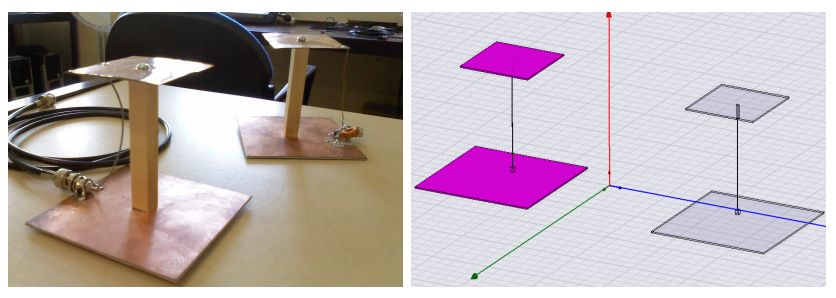

Figure 12: Test electrodes. 
circuit of Figure 6.

It is to be noted that, while one of the load capacitances $C_{L}$ (corresponding to the emitter electrode) will be fed by the applied signal, the corresponding to the receiver electrode will be connected in parallel with the coaxial cable and oscilloscope's input amplifier capacitances which added about $200 \mathrm{pF}$ (the simulated $C_{L}$ was $5.5 p F$ ). In order to minimize these external added capacitances, tests with a low input capacitance $(3 \mathrm{pF})$ buffer amplifier were also done. The results are presented in Figure 13 and are labeled A and B for the unbuffered and buffered cases, respectively. Naturally, the loading effect was also included in the simulations and in the theoretical model.

Figure 13 shows that theory has the most optimistic results, followed by simulations. But in most of the cases the error is less than $5 \mathrm{~dB}$ and always well under $20 \mathrm{~dB}$ (or one order of magnitude). It seems that the effect of the presence of the external load is, in fact, worse than its additive effect, probably because it can change substancialy the coupling capacitance too. Another possible reason for the differences has to do with the faraday cage and the thin wires connecting the electrodes to the connectors, which were not considered in the theoretical model (but were aproximately included in the simulations). Indeed, if only simulation and the experimental results are compared, the maximum difference is always lower than $5 \mathrm{~dB}$ which is a relatively good match for a channel gain and range prediction and warrants the accuracy of the simulators and the simulation's methodology.

It is interesting to note that the results in Figure 13 compare well with value presented in [4] as the worst case for capacitive coupling communications for an average human (and reasonable sized electrodes) - $80 \mathrm{~dB}$, which demonstrated that the distance between electrodes can be larger than that indicated by [3].

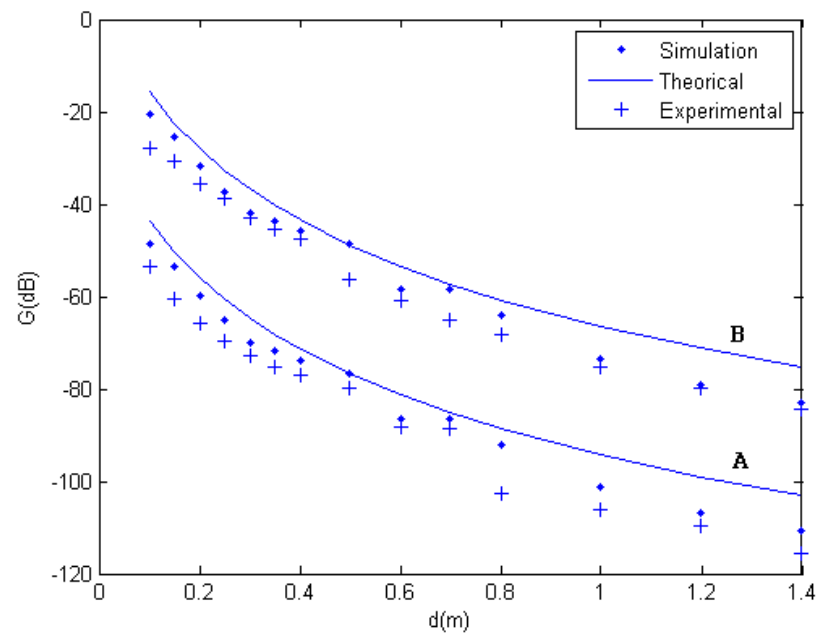

Figure 13: Pratical experiments and simulations: A: receiver electrode directly connected to oscilloscope by coaxial cable; B: receiver electrode buffered with a $3 \mathrm{pF}$ input capacitance amplifier.
As a proof of concept, a simple pair of electrodes and electronics were build to transmit a square $3 \mathrm{Vpp}, 10 \mathrm{kHz}$ signal using capacitive coupling communications. Frequency Shift Keying (FSK) modulation was used (with a central frequency of $10.7 \mathrm{MHz}$ ). Even with crude electronics was possible to recover the $10 \mathrm{kHz}$ signal at a $30 \mathrm{~cm}$ distance (between emitter and receiver electrodes), even when the electrodes were placed in different subjects shaking hands (see Figure 14). The receiver has an electrical to optical converter and it is connected to the oscilloscope via an optical fiber (of course at the oscilloscope side there is an optical to electrical converter). This setup isolates the oscilloscope from the communication system as explained before.

\section{CONCLUSIONS}

A simple theoretical model for the channel gain of a capacitance coupled communication system has been presented. Overall, it was found a significant coherence between the proposed theoretical model, the simulations and the experimental results. We have seen that theoretical model approximates the practical results well within an order of magnitude and therefore can be used to predict the working range of similar systems.

As expected the values of the coupling capacitances are extremely low when compared to the electrode's capacitances. This implies that the "emitted" signal is strongly attenuated and to cover all the body very sensitive receivers must be used.

Another important conclusion is that a good input buffer is very important for this kind of communications, as figure 13 gives much better results for the B case (the buffered case).

For future work, research about replacing the human body as conductive plane by a more realistic human body "wire" must be performed. This approximation must also be further investigated regarding the position of the electrodes in the body: probably an arm will not behave so close to an "infinite ground plane" as the chest. This study should also consider the effects of increasing the frequency well beyond $10 \mathrm{MHz}$.

\section{Acknowledgment}

We would like to thank Prof. Armando Rocha of DETI. This work is supported by FCT - Fundação para a Ciência e Tecnologia, Grant: SFRH_BD_41808_2007.
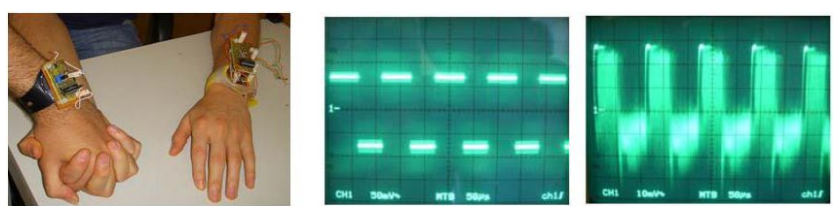

Figure 14: Practical system (Emitted signal vs. Received signal). 


\section{REFERENCES}

[1] João Henrique Donker, "The body as a communication medium", Department of EMCS, University of Twente, $11^{\text {th }}$ Twente Student Conference on IT, Enschede $29^{\text {th }}$ June, 2009.

[2] Ecma International: Standard ECMA-340, Near Field Communication Interface and Protocol (NFCIP-1), December 2004, http://www.ecmainternational.org/publications/standards/Ecma-340.htm. Retrieved 30 March 2012

[3] Thomas Guthrie Zimmerman, "Personal Area Networks (PAN): Near-Field Intra-Body Communication", Massachusetts Institute of Technology, September 1995.

[4] Nafiseh Seyed Mazloum, "Body-Coupled Communications: Experimental characterization, channel modeling and physical layer design", Chalmers University of Technology Philips Research: Department of Signals and Systems Distributed Sensor Systems, December 2008.

[5] M.Shingawa, M.Fukumoto, K.Ochiai, and H.Kyuragi. A, "Near-field-sensing transceiver for intrabody communication based on the electrooptic effect", IEEE Transactions on instrumentation and measurement, 53(6), Dec. 2004.

[6] Yong Song, Qun Hao et al., "The Simulation Method of the Galvanic Coupling Intrabody Communication With Different Signal Transmission Paths", IEEE Transactions on Instrumentation and Measurement, Vol. 60, No. 4, April 2011.

[7] Lin Zhong, Dania El-Daye et al., "OsteoConduct: Wireless Body-Area Communication based on Bone Conduction", Dept. of Electrical and Computer Engineering, Dept. of Bioengineering of Rice University.

[8] Stefan Drude, "Requirements and Application Scenarios for Body Area Networks", NXP Semiconductors, Eindhoven, The Netherlands.

[9] Hoi-Jun Yoo et al., "Low Energy On-Body Communication for BSN", in Proc. IFMBE, Springer, 2007.

[10] Namjun Cho, Seong-Jun Song et al., "The Human Body Characteristics as a Signal Transmission Medium for Intrabody Communication", IEEE Transactions on Microwave Theory and Techniques, Vol. 55, No. 5, May 2007.

[11] Marc Simon Wegmueller, Andreas Kuhn et al., "An Attempt to Model the Human Body as a Communication Channel", IEEE Transactions on Biomedical Engineering, Vol. 54, No. 10, October 2007.

[12] K. Hachisuka, A. Nakata, T. Takeda, Y. Terauchi, K. Shiba, K. Sasaki, H. Hosaka and K. Itao, "Development and performance analysis of an intra-body communication device", In Proc. TRANSDUCERS, Solid-State Sensors, Actuators and Microsystems, 12th International Conference on, volume 2, pages 1722-1725, 8-12 June 2003.

[13] Chao Liu, Mickel Budhia et al., "A Generalized Coupling Model for Capacitive Power Transfer
Systems".

[14] Kip, Arthur F., Fundamentals of electricity and magnetism, McGraw-Hill Book, New York, 1962.

[15] Inan, Umran S., Engineering electromagnetics, Addison-Wesley, Menlo Park (CA), 1999.

[16] Feynman, Leighton, Sand, The Feynman Lectures on Physics, Addison-Wesley, 1977.

[17] Kraus, John D., Antennas, MC-Graw Hill, New York, 1988.

[18] Wikipedia, The free encyclopedia, Online: www.wikipedia.com Retrieved 20 September 2011.

[19] Ansoft HFSS, Electromagnetic Simulator, Online: www.ansoft.com Retrieved 10 January 2012.

[20] SAP - Smart Analysis Program, Circuit Interconnection Parameter Extration, Online: www.iue.tuwien.ac.at , Retrieved 10 January 2012. 